\title{
Winde und Zyklonen
}

Joaquim G. Pinto, Mark Reyers

8.1 Gegenwärtiges Klima und beobachtete Trends - 68

8.2 Trends im zukünftigen Klima - 69

8.3 Kurz gesagt -73

Literatur - 73 
Stärkere gerichtete Bewegungen der Luft werden als Wind bezeichnet. Sie entstehen durch Unterschiede des Luftdrucks in der Erdatmosphäre, wobei die Luft immer von Gebieten mit hohem Druck in Richtung des tiefen Drucks bewegt wird. Durch die Erdrotation wird die Luft auf der Nordhalbkugel zusätzlich nach rechts relativ zur Strömungsrichtung abgelenkt, sodass der großskalige Wind parallel zu Bereichen mit gleichem Druck weht. Der großskalige Wind stellt sich annähernd in der Höhe ein, wo sich die Kräfte, die aufgrund des Druckgradienten und der Erdrotation wirken, im Gleichgewicht befinden und es keinen Einfluss der Bodeneigenschaften gibt. Der Großteil Europas befindet sich in den mittleren Breiten, wo im Mittel der Druck von Süden nach Norden hin abnimmt. Damit liegen weite Teile Europas und speziell Deutschland in einem Bereich, in dem der mittlere Wind aus Westen kommt. Die Stärke der Westwinde über Europa wird vor allem durch den Druckunterschied zwischen den niederen und höheren Breiten über dem östlichen Nordatlantik bestimmt: Je stärker der Druckunterschied zwischen Azorenhoch und Islandtief ist, desto stärker ist der großskalige Wind. Der Druckunterschied zwischen subtropischen und subpolaren Luftmassen ist im Winter am größten. Deshalb ist in der Regel der großskalige Wind im Winter stärker als im Sommer.

Der lokale Wind kann sich durch den Einfluss von Bodeneigenschaften, Höhenstrukturen, atmosphärischen Bedingungen und lokalen Gegebenheiten stark vom großskaligen Wind unterscheiden. Dabei sind Böen - also kurzfristige Abweichungen vom Mittelwind - von besonderer Bedeutung, da sie deutlich höhere Geschwindigkeiten aufweisen als der mittlere Wind. Böen können beispielsweise auftreten, wenn Luftströmungen aus größeren Höhen, die meist höhere Windgeschwindigkeiten besitzen als bodennahe Luftströmungen, durch atmosphärische Turbulenzen Richtung Erdboden transportiert werden.

Die stärksten Winde und Böen in Nord- und Zentraleuropa treten in Verbindung mit Zyklonen der mittleren Breiten auf - Tiefdruckwirbel mit einem Durchmesser von bis zu einigen Tausend Kilometern. Im Zentrum von Zyklonen herrschen typischerweise tiefe Luftdruckwerte von $970-1000 \mathrm{hPa}$, in manchen Extremfällen können diese auch unterhalb von $920 \mathrm{hPa}$ liegen. Aufgrund der oben genannten Kräfte kann es dadurch zu einer starken Bewegung von Luftmassen mit einer Geschwindigkeit von bis zu $200 \mathrm{~km} / \mathrm{h}$ entgegen dem Uhrzeigersinn um das Zyklonenzentrum kommen. Dabei werden Winde mit einer Stärke von mindestens 9 Beaufort (ca. $75 \mathrm{~km} / \mathrm{h}$ ) bereits als Sturm bezeichnet. Somit beeinflussen Zyklonen maßgeblich die Winde in den mittleren Breiten und tragen außerdem in erheblichem Maße zu den Witterungs- und klimatischen Bedingungen in Europa bei: Zum einen sind sie für den Transport von Feuchte und Wärme nach Europa verantwortlich und bestimmen somit das Klima in Deutschland. Zum anderen sind Zyklonen für einen Großteil von extremen Wetterereignissen wie Starkniederschläge, Sturmböen und Überflutungen bzw. Sturmfluten in den mittleren Breiten verantwortlich (Ulbrich et al. 2009; Schwierz et al. 2010), die auch in Deutschland zu erheblichen Schäden führen können. Ein prominentes Beispiel ist der Sturm Kyrill (Fink et al. 2009), der vom 17. bis 19.01.2007 über Mitteleuropa zog, Dutzende Todesopfer forderte sowie erhebliche Forst- und Gebäudeschäden verursachte. Weitere Beispiele aus der jüngeren Vergangenheit sind die Stürme Paula und Emma (beide Anfang 2008) sowie Xynthia (Februar 2010), Christian (Oktober 2013) und Xaver (Dezember 2013).

Zyklonen entstehen in Regionen mit hohen Temperaturunterschieden, indem sie Energie, die durch die Hebung von Luftpaketen aufgebaut wird (z. B. durch Erwärmung), in Bewegungsenergie in Form von Wind umwandeln. Diese ausgeprägten Temperaturgradienten können zum einen durch die unterschiedlich starke solare Erwärmung niedriger und hoher Breiten entstehen, oder sie bilden sich aufgrund der unterschiedlich starken Erwärmung von Land- und Meeresoberflächen. Die besten Bedingungen für das Entstehen und die weitere Entwicklung von Zyklonen herrschen über dem Nordatlantik - besonders über dem westlichen Nordatlantik in der Nähe von Neufundland, wo beide genannten Effekte zur Bildung von Temperaturgradienten gegeben sind. Die sich entwickelnden Zyklonen wandern anschließend mit der westlichen Grundströmung nach Europa, wo sie meistens Richtung Britische Inseln und Skandinavien weiterziehen. Gemessen an der Gesamtzahl der Zyklonen treffen hingegen vergleichsweise wenige auf das Festland Westeuropas. Insgesamt werden ihre Zugbahnen stark von den oben genannten Druckunterschieden zwischen Azorenhoch und Islandtief beeinflusst. Im Falle eines stark ausgeprägten Azorenhochs und Islandtiefs werden Zyklonen hauptsächlich Richtung Skandinavien abgelenkt, während sie bei einem schwach ausgeprägten Azorenhoch und Islandtief auch weiter südlich auf das europäische Festland treffen können (Pinto et al. 2009). Das sich über dem Nordatlantik befindliche Gebiet, in dem vermehrt Zyklonen entstehen und sich entwickeln, wird auch nordatlantischer storm track genannt (Hoskins und Valdes 1990). Der Begriff steht in diesem Zusammenhang für die mittleren Zugbahnen von Hoch- und Tiefdruckgebieten. Die storm tracks bilden daher ein geeignetes Maß zur Bewertung der Auswirkung des Klimawandels auf die für Europa und Deutschland relevanten Zyklonen. So geht eine mögliche Verlagerung dieser storm tracks in den vergangenen Jahrzehnten und in einem zukünftigen Klima also mit veränderten Zugbahnen der Zyklonen und somit veränderten klimatischen Bedingungen und bodennahen Winden über Deutschland einher.

In den vergangenen Jahren wurden verschiedene objektive Verfahren zur Identifizierung von Zyklonen sowie deren Zugbahnen in Reanalysen und globalen Klimamodellen (global climate models) entwickelt. Dabei hat sich gezeigt, dass sich die Ergebnisse dieser Verfahren stark unterscheiden können. So ist die Identifizierung von Zyklonen nicht nur sensitiv gegenüber der Wahl des Verfahrens an sich, sondern auch gegenüber den Eingangsdaten, auf die das Verfahren angewendet wird (Raible et al. 2008; Ulbrich et al. 2009; Neu et al. 2013). Diese Unsicherheiten sollten bei der Bewertung von Zukunftsszenarien berücksichtigt werden.

\subsection{Gegenwärtiges Klima und beobachtete Trends}

Deutschland ist im gegenwärtigen Klima durch regional unterschiedliche Windgeschwindigkeiten geprägt. Im klimatologi- 
schen Mittel ist der Wind im küstennahen Bereich am stärksten. Mit zunehmendem Abstand von der Küste ist ein deutlicher Rückgang der mittleren Windgeschwindigkeit zu verzeichnen. Ausnahmen bilden die höheren Lagen wie z. B. der Nordrand der Alpen oder die Mittelgebirge, wo im Durchschnitt höhere Windgeschwindigkeiten auftreten. Im Gegensatz dazu herrschen in Tallagen - etwa im Rheintal - niedrigere mittlere Windgeschwindigkeiten vor.

Die Böengeschwindigkeiten zeigen ein ähnliches Muster wie der mittlere Wind, mit hohen Werten über dem Meer und einer Abnahme landeinwärts (• Abb. 8.1). In Tallagen sind die Böengeschwindigkeiten besonders niedrig, so z. B. im Rhein- oder Donautal. Eine besonders heterogene Verteilung der Wind- und Böengeschwindigkeit ist in Gebieten mit komplexen Höhenstrukturen zu finden, etwa im Schwarzwald.

Die Windverteilungen in Deutschland, speziell das Nord-SüdGefälle der Wind- und Böengeschwindigkeiten, sind entscheidend von der Stärke und den Zugbahnen der vom Nordatlantik kommenden Zyklonen geprägt (• Abb. 8.2). Während eine große Anzahl von ihnen über den Bereich der Nordsee zieht und damit für starke Windverhältnisse in den Küstenregionen sorgt, wird der Süden Deutschlands seltener von starken Zyklonen getroffen.

Da die storm tracks stark von den Temperaturverteilungen im Nordatlantikbereich abhängen, ist zu erwarten, dass der Klimawandel zu einer Veränderung der storm tracks und somit der Zyklonenaktivität führt und sich damit die Windverhältnisse über Deutschland ändern. Studien zu historischen Trends dieser Aktivität liefern jedoch unterschiedliche Aussagen (für eine Literaturübersicht s. Ulbrich et al. 2009 oder Feser et al. 2015). Die meisten Studien, die auf Reanalysen für die zweite Hälfte des 20. Jahrhunderts beruhen, zeigen eine generelle Zunahme der nordatlantischen storm track-Aktivität (Chang und Fu 2002; $\mathrm{Hu}$ et al. 2004). In einigen Studien wird für längere Zeiträume eine Zunahme der Zyklonenanzahl über dem Nordatlantik identifiziert (z.B. Wang et al. 2009, 2011; Schneidereit et al. 2007), während Hanna et al. (2008) und Lambert (2004) einen Rückgang feststellen. Für Europa wiederum ergeben sich sehr heterogene Trends in Bezug auf die beobachtete Anzahl an Zyklonen: So fand Trigo (2006) für den Zeitraum von 1958 bis 2002 eine Zunahme der Zyklonenanzahl über Nordeuropa, aber eine Abnahme über Mittel- und Südeuropa heraus. Einen generellen Anstieg der Anzahl von starken Zyklonen über dem östlichen Nordatlantik und der südlichen Nordsee seit 1958 haben Weisse et al. (2005) gefunden. Dieser Trend hat sich jedoch gegen Ende des 20. Jahrhunderts über der Nordsee stark abgeschwächt und über dem Nordatlantik sogar umgekehrt. Bestätigt werden diese Ergebnisse von Druckmessungen an Stationen (Schmidt und von Storch 1993). Diese Stationsmessungen ermöglichen ebenfalls eine Abschätzung des großskaligen Windes und haben den Vorteil, dass sie viel weiter in die Vergangenheit reichen als Reanalysen. Alexandersson et al. (2000) konnten zeigen, dass der positive Trend seit den 1950er-Jahren und die Abschwächung zum Ende des 20. Jahrhunderts auch in den Stationsdaten vorhanden sind. Die Betrachtung eines weitaus länger zurückreichenden Zeitraums in den Stationsmessungen weist allerdings auch darauf hin, dass diese Schwankungen innerhalb der natürlichen Variabilität des Sturmklimas liegen.
Feser et al. (2015) haben die Ergebnisse von Studien zusammengefasst, die sich ausschließlich mit Stürmen über dem Nordatlantik und Europa befassen, und sind zu folgendem Schluss gekommen: Während Studien, die auf Daten aus Stationsmessungen basieren, für Mitteluropa und die Nordsee häufig eine Abnahme der Sturmaktivität im 20. Jahrhundert zeigen, weisen Reanalysen auf keinen oder einen positiven Trend hin. Der aktuelle Fünfte Sachstandsbericht des Weltklimarates (IPCC AR5) wiederum weist auf einen Trend zu mehr und intensiveren Zyklonen über dem Nordatlantik in Reanalysen der vergangenen 60 Jahre hin (Hartmann et al. 2013). Ein Grund für diese teils widersprüchlichen Aussagen liegt in den unterschiedlichen Verfahrensweisen, die bei diesen Studien verwendet werden. Wichtige Faktoren sind dabei die verschiedenen Verfahren zur Quantifizierung der Zyklonenaktivität und unterschiedliche Datensätze (Raible et al. 2008; Neu et al. 2013). Ein weiteres Problem liegt in dem begrenzten Zeitraum von ungefähr 50 Jahren, für den flächendeckende Beobachtungen vorliegen. So ist es schwierig festzustellen, ob eine beobachtete Änderung in diesem Zeitraum einem langzeitlichen Trend entspricht oder auf Zeitskalen von einzelnen oder mehreren Dekaden innerhalb der natürlichen Variabilität liegt, die für die Zyklonenaktivität sehr ausgeprägt ist (Donat et al. 2011b; Krueger et al. 2013; Wang et al. 2011). Die geringere Dichte von Beobachtungsdaten vor den 1960erJahren trägt zusätzlich zu diesen Unsicherheiten bei. Wie oben erwähnt, sind jedoch sehr lange Beobachtungsreihen erforderlich, um verlässliche Aussagen über die tatsächliche Veränderung des Sturmklimas über Deutschland treffen zu können (Bärring und von Storch 2004; Matulla et al. 2008). Entsprechend kann für Deutschland ebenfalls kein klarer Trend der Zyklonenaktivität gefunden werden, da auch hier die zwischenjährlichen und dekadischen Schwankungen weitaus stärker sind als ein möglicher langzeitlicher Trend.

Die oben genannten Schlussfolgerungen bezüglich der Unsicherheit der beobachteten Zyklonenaktivität innerhalb der letzten Jahrzehnte gelten auch für die beobachteten Windverhältnisse über Europa und Deutschland (IPCC AR5; Hartmann et al. 2013). Wang et al. (2011) z. B. zeigen für die Nordsee und die Alpen eine Zunahme für das Auftreten starker Winde bis Ende des 20. Jahrhunderts. Allerdings hat sich dieser positive Trend über der Nordsee seit Mitte der 1990er-Jahre abgeschwächt. Insgesamt ist somit für Deutschland in Bezug auf Zyklonen und Winde kein eindeutiger historischer Langzeittrend zu finden (Hofherr und Kunz 2010). Dies steht im Einklang mit Studien, die sich mit dem Windstauklima über der Nordsee - also der Veränderung des Wasserspiegels durch Windeinfluss - und den damit verbundenen Sturmfluten befassen ( Kap. 9). Auch das Windstauklima zeigt ausgeprägte dekadische Schwankungen, aber keinen erkennbaren historischen Trend.

\subsection{Trends im zukünftigen Klima}

Das World Climate Research-Programm hat die sogenannten Coupled Model Intercomparison-Projekte (CMIP) ins Leben gerufen, um eine umfassende Evaluierung von verschiedenen Klimamodellen und Klimamodellprojektionen zu ermöglichen. 


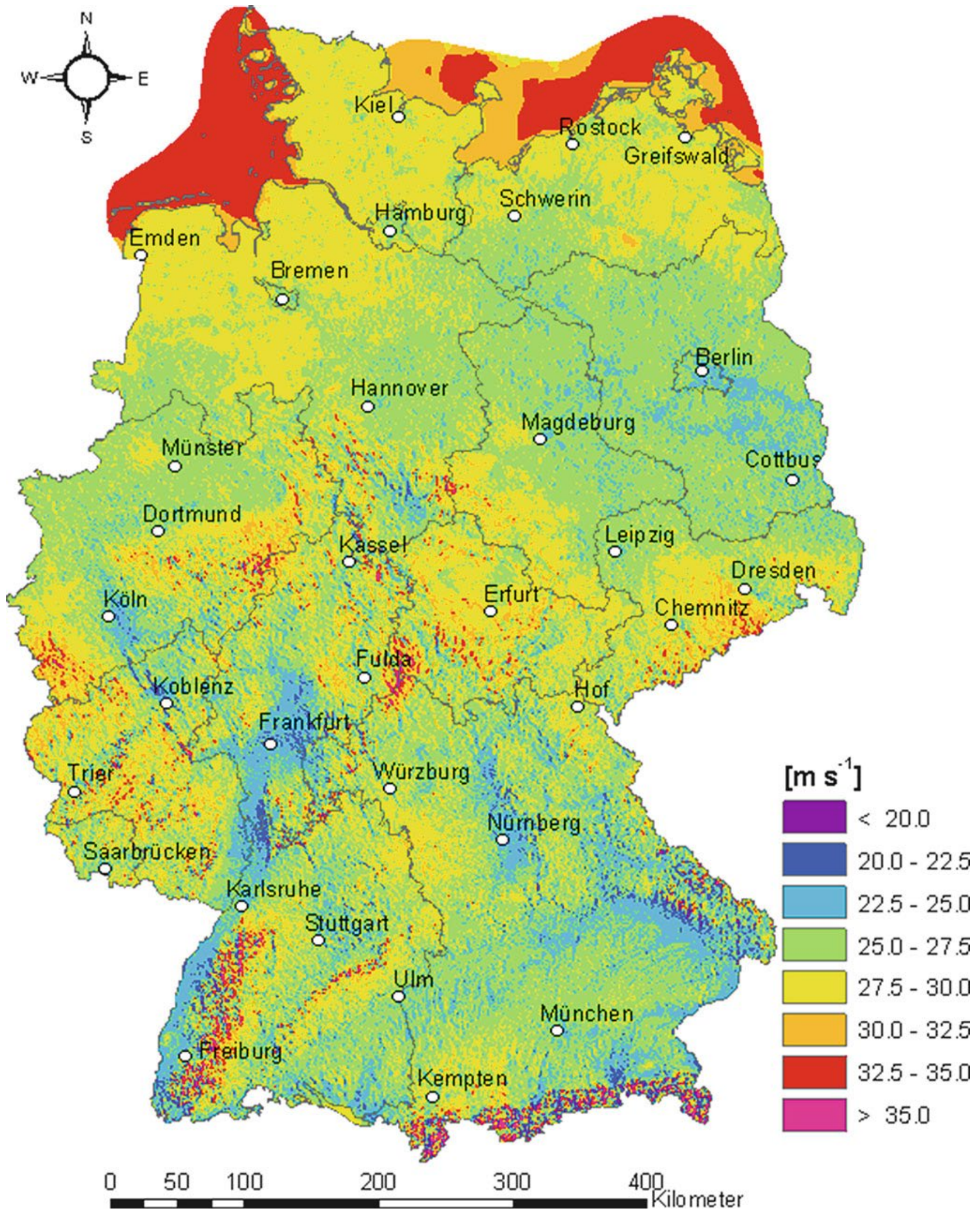

- Abb. 8.1 Simulierte maximale Böengeschwindigkeit über Deutschland mit einer Wiederkehrperiode von 2 Jahren für den Zeitraum 1971-2000 mit einer Auflösung von $1 \times 1 \mathrm{~km}$. (Aus Hofherr und Kunz 2010) 


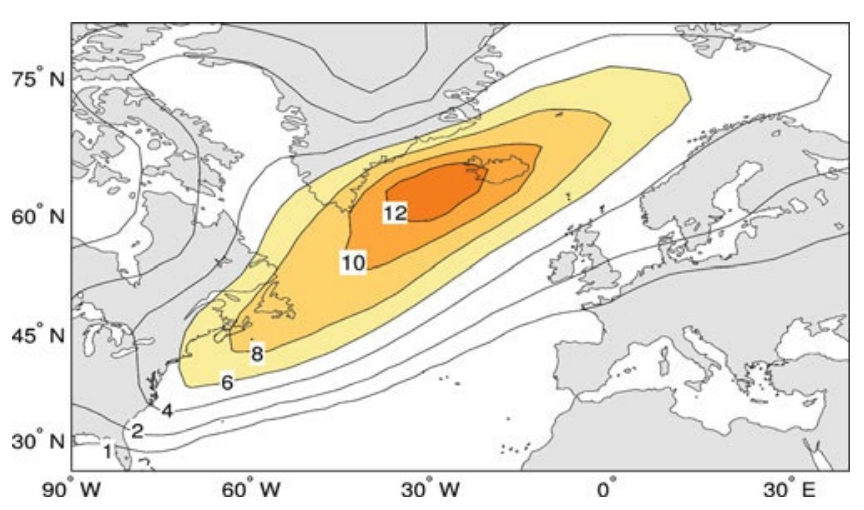

- Abb. 8.2 Flächengewichtete Dichte der Zyklonenzugbahnen von starken Zyklonen (in Anzahl der Tage mit Zyklonen pro Winter pro Breitengrad zum Quadrat) für den Zeitraum 1958-1998, abgeleitet aus den stärksten $10 \%$ aller Zyklonen. (Aus Pinto et al. 2009)

Eine Vielzahl von Modellen der beiden aktuellsten CMIP-Projekte (CMIP3 und CMIP5) simulieren im Mittel für die Nordhemisphäre eine Nordverschiebung der Zyklonenzugbahnen bzw. storm tracks bis Ende des 21. Jahrhunderts (Yin 2005; Gastineau und Soden 2009; Harvey et al. 2012). Des Weiteren herrscht eine gute Übereinstimmung bezüglich einer generellen Abnahme der Anzahl aller Zyklonen im globalen Mittel. Große Unsicherheiten gibt es hingegen im Hinblick auf mögliche regionale Änderungen der Zyklonenaktivität (Ulbrich et al. 2008, 2009) sowie den damit verbundenen zukünftigen Trends der regionalen Charakteristika von Windböen und Böen. Diese Unsicherheiten basieren hauptsächlich auf einer von Klimamodellen unterschiedlich projizierten Veränderung der Temperaturgradienten zwischen den Subtropen und der Polarregion in der oberen und unteren Troposphäre, also jenem Teil der Atmosphäre, der je nach Klimazone bis in eine Höhe von ungefähr $8-15 \mathrm{~km}$ reicht (Harvey et al. 2014). Zusätzlich beeinflussen lokale, nur schwer vorhersagbare Prozesse die regionale Änderung der storm-trackAktivität (IPCC AR5, Kirtman et al. 2013). In einigen Studien wird beispielsweise ein Einfluss des Meereisrückgangs auf die Zyklonenaktivität nachgewiesen (Bader et al. 2011; Deser et al. 2010). Für Zyklonen über dem Nordatlantik spielen vermutlich Änderungen in der Ozeanzirkulation eine wichtige Rolle, die in den verschiedenen Klimamodellen teils sehr unterschiedlich wiedergegeben werden (Woollings et al. 2012).

Einige Klimaprojektionen anhand des Ensembles aus dem Programm CMIP3 deuten auf eine Ausdehnung des nordatlantischen storm tracks nach Osten hin und damit auf eine Verschiebung der Zyklonenzugbahnen in Richtung Europa (Bengtsson et al. 2006, 2009; Catto et al. 2011; Pinto et al. 2007b; Ulbrich et al. 2008). Auch in CMIP5-Modellen zeigt sich eine solche Verschiebung Richtung Europa, die hier jedoch schwächer ausgeprägt ist (Harvey et al. 2012; Zappa et al. 2013). Des Weiteren ist anzunehmen, dass es in einer wärmeren Atmosphäre aufgrund von mehr verfügbarer latenter Wärme, die beim Phasenübergang von Wasserdampf zu Flüssigwasser frei wird, zu besseren Wachstumsbedingungen für starke Zyklonen kommen kann und somit zu potenziell stärkeren Stürmen (Pinto et al. 2009; Fink et al. 2012). Eine deutliche Erhöhung der Sturmaktivität über Westeuropa wäre die Folge (Pinto et al. 2009; Donat et al. 2010; McDonald 2011). Dies stimmt mit kürzeren Wiederkehrperioden von starken Zyklonen über der Nordsee und Westeuropa bis zum Jahr 2100 überein, wie sie Della-Marta und Pinto (2009) gefunden haben. Insgesamt zeigt der größte Teil der Studien, die sich mit der Sturmaktivität befassen, eine Zunahme der Anzahl von Stürmen über Mitteleuropa und der Nordsee bis zum Ende des 21. Jahrhunderts (für eine Literaturübersicht s. Feser et al. 2015).

Die meisten Studien und Modelle stimmen darin überein, dass es durch die Zunahme starker Zyklonen insgesamt zu einem häufigeren Auftreten von Starkwindereignissen kommt. Dies wurde sowohl in globalen als auch regionalen Modellen mit Fokus auf Deutschland festgestellt. So simulieren einige globale CMIP3-Klimamodelle für das Ende des 21. Jahrhunderts stärkere maximale tägliche Windgeschwindigkeiten über Nordwesteuropa, der Nordsee und Deutschland (Pinto et al. 2007a; Donat et al. 2010) oder auch mehr Starkwindereignisse über Nordeuropa (Gastineau und Soden 2009). Studien auf Basis von CMIP5-Klimamodellen kommen zu dem Schluss, dass die Anzahl von Starkwindereignissen im Zusammenhang mit Zyklonen über Mitteleuropa steigen kann (Zappa et al. 2013). Auf der regionalen Skala gibt es einige übereinstimmende Ergebnisse vor allem für den nördlichen Bereich Zentraleuropas (Beniston et al. 2007; Rockel und Woth 2007; Fink et al. 2009; Hueging et al. 2013). So identifizieren Rockel und Woth (2007) anhand eines Ensembles von Regionalmodellen für den Zeitraum 2071-2100 eine Zunahme der täglichen maximalen Windgeschwindigkeiten in Mitteleuropa im Winter, während im Herbst eine Abnahme festzustellen ist. Ein negativer Trend bis zum Ende des 21. Jahrhunderts für die höchsten Wind- bzw. Böengeschwindigkeiten über Deutschland findet sich in verschiedenen hoch aufgelösten Modellen auch für den Sommer (Bengtsson et al. 2009; Hueging et al. 2013; Walter et al. 2006). Der Fünfte Sachstandsbericht des IPCC kommt zu der Schlussfolgerung, dass es bis zum Ende des 21. Jahrhunderts im Winter zu einer leichten Zunahme von Starkwinden über Nord- und Mitteleuropa kommen wird, während keine verlässlichen Trends für andere Jahreszeiten und Regionen gefunden werden (Kovats et al. 2014). Folglich wird in den meisten Klimamodellen für Nord- und Mitteleuropa eine Zunahme des Windes und somit des Windenergieertrags im Winter erwartet (Hueging et al. 2013), wobei sich die Stärke dieser Zunahme in den verschiedenen Klimamodellen deutlich unterscheiden kann (Reyers et al. 2016). Die Projektionen für den jährlichen Windenergieertrag über Deutschland variieren hingegen je nach Modell zwischen einer Ab- und Zunahme von bis zu $10 \%$ (Tobin et al. 2015). Für Deutschland zeigen die meisten Regionalisierungen insgesamt einen generellen Anstieg der Böengeschwindigkeit im Norden und Nordwesten sowie an der Nord- und Ostseeküste (Walter et al. 2006; Rauthe et al. 2010). Speziell für Nordrhein-Westfalen zeigt sich ein deutlicher Anstieg der Böengeschwindigkeit zum Ende des 21. Jahrhunderts (Pinto et al. 2010).

Ein besonderer Fokus wird auf eine Studie von Rauthe et al. (2010) gelegt, da in dieser Untersuchung Ergebnisse von sieben verschiedenen regionalen Klimamodellen für die Projektion von Böengeschwindigkeiten berücksichtigt werden und nicht nur von einzelnen Modellen wie in der Mehrzahl anderer Studien. Dabei zeigt das Mittel über alle sieben Modelle (Ensemblemittel) eine Zunahme bis 2050 von bis zu $7 \%$ für Böen mit einer Wieder- 


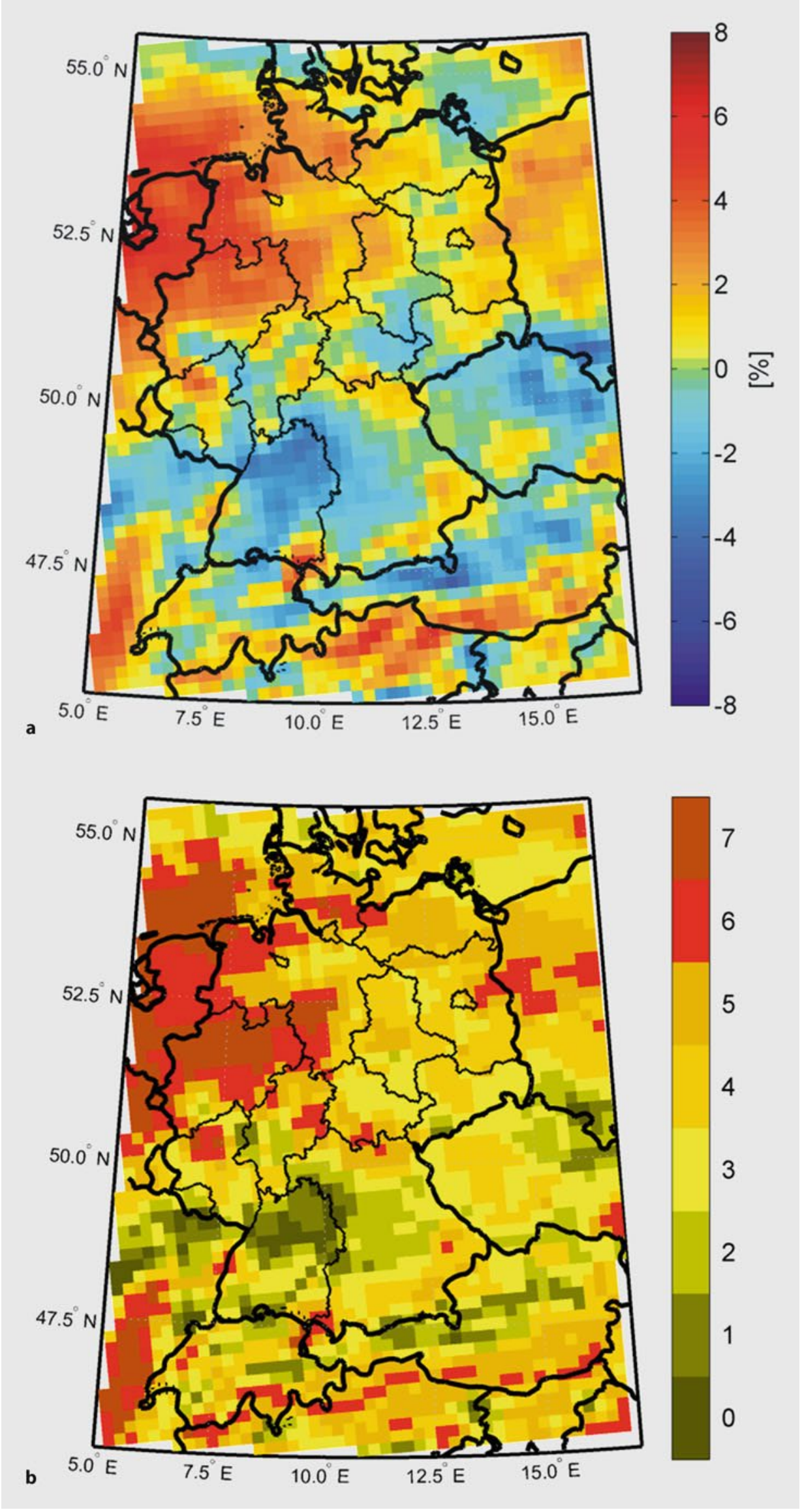

- Abb. 8.3 Ensemblemittel von sieben regionalen Klimamodellen ( $R C M)$ : a Relative Änderung der mittleren Böe mit einer Wiederkehrperiode von 10 Jahren für den Zeitraum 2021-2050 im Vergleich zu 1971-2000, b Anzahl der RCM-Simulationen mit einer positiven relativen Änderung der Böengeschwindigkeit mit einer Wiederkehrperiode von 10 Jahren. (Rauthe et al. 2010) 
kehrperiode von 10 Jahren über Teilen Nordrhein-Westfalens und Niedersachsens ( Abb. 8.3a). Zusätzlich stimmen für diesen Bereich nahezu alle Modelle dahingehend überein, dass es generell zu einer Zunahme starker Böen kommt (• Abb. 8.3b). Große Übereinstimmung zwischen den Modellen findet sich außerdem für den Norden Baden-Württembergs, wo bis zu sieben Modelle eine Abnahme simulieren (- Abb. 8.3b), die im Ensemblemittel mehr als $-6 \%$ betragen kann ( $\bullet$ Abb. 8.3a). Für andere Bereiche Deutschlands sind die Ergebnisse hingegen nicht eindeutig. So projiziert das Ensemblemittel z. B. für Schleswig-Holstein einen Anstieg starker Böen um 2-6\% (• Abb. 8.3a), jedoch simulieren teilweise nur vier von sieben Modellen eine generelle Zunahme (- Abb. 8.3b). Insgesamt lässt sich schlussfolgern, dass abgesehen von einigen wenigen Bereichen in Deutschland bezüglich der möglichen zukünftigen $\mathrm{Zu}$ - oder Abnahme von starken Böen Unsicherheit zwischen den einzelnen Modellen herrscht.

Die projizierte Zunahme von Starkwindereignissen und Böengeschwindigkeiten, vor allem im Winter, hätte einen Anstieg der potenziellen Gebäudeschäden im Zusammenhang mit Winterstürmen über Mitteleuropa zur Folge, mit starken Auswirkungen für Deutschland (Schwierz et al. 2010; Donat et al. 2011a; Pinto et al. 2012; Held et al. 2013). Für eine detaillierte Analyse der ökonomischen Auswirkungen des Klimawandels sei hier auf - Kap. 25 verwiesen.

\subsection{Kurz gesagt}

In Beobachtungen der vergangenen Jahrzehnte und in Klimaprojektionen für das zukünftige Klima wird eine starke zwischenjährliche Variabilität der Zyklonenaktivität über dem Nordatlantik festgestellt. Unsicherheit herrscht dagegen über einen langzeitlichen Trend der Zyklonenanzahl und -intensitäten, vor allem in Regionen des europäischen Festlands. So zeigt sich in Reanalysedaten für die zweite Hälfte des 20. Jahrhunderts eine ausgeprägte dekadische Variabilität der Zyklonenaktivität über dem östlichen Nordatlantik, Europa, Deutschland und der Nordsee. Druckmessungen an Stationen über Nordeuropa und Deutschland belegen eine starke derartige Variabilität sogar für einen noch längeren Zeitraum. Ein langzeitlicher Trend kann jedoch nicht verlässlich identifiziert werden. Dasselbe gilt für die Windverhältnisse über Deutschland in den vergangenen 50 Jahren.

Für das zukünftige Klima ist eine Verschiebung des nordatlantischen storm tracks in Richtung Europa wahrscheinlich, was jedoch nicht durch eine Zunahme der Gesamtzahl aller Zyklonen, sondern durch ein häufigeres Auftreten starker Zyklonen bedingt ist. Die Wiederkehrperiode starker Zyklonen über der Nordsee und Westeuropa wird sich demnach verkürzen, während es bis 2100 allgemein weniger Zyklonen geben wird. Daher ist es wahrscheinlich, dass bereits ab Mitte des 21. Jahrhunderts mehr Starkwindereignisse und starke Böen über der Nordsee und Nordwestdeutschland auftreten werden. Diese werden vor allem im Winter zunehmen, während es im Sommer eher zu einer Abnahme kommen wird. Für die anderen Bereiche Deutschlands sind Aussagen für zukünftige Klimatrends in Bezug auf den Wind unsicher; es werden aber nur geringe Änderungen im Vergleich zum gegenwärtigen Klima erwartet.

\section{Literatur}

Alexandersson H, Tuomenvirta H, Schmith T, Iden K (2000) Trends of storms in NW Europe derived form an updated pressure data set. Climate Research 14:71-73

Bader J, Mesquita MDS, Hodges KI, Keenlyside N, Osterhus S, Miles M (2011) A review on Northern hemisphere sea-ice, storminess and the North Atlantic Oscillation: Observations and projected changes. Atmospheric Research 101:809-834

Bärring L, von Storch H (2004) Scandinavian storminess since about 1800. Geophysical Research Letters 31:L20202

Bengtsson L, Hodges Kl, Roeckner E (2006) Storm tracks and climate change. Journal of Climate 19(15):3518-3543

Bengtsson L, Hodges KI, Keenlyside N (2009) Will Extratropical Storms Intensify in a Warmer Climate? Journal of Climate 22:2276-2301

Beniston M, Stephenson DB, Christensen OB, Ferro CAT, Frei C, Goyette S, Halsnaes K, Holt T, Jylha K, Koffi B, Palutikof J, Scholl R, Semmler T, Woth K (2007) Future extreme events in European climate: an exploration of regional climate model projection. Climatic Change 81:71-95

Catto JL, Shaffrey LC, Hodges KI (2011) Northern hemisphere extratropical cyclones in a warming climate in the HiGEM high resolution climate model. Journal of Climate 24:5336-5352

Chang EKM, Fu Y (2002) Interdecadal variations in Northern hemisphere winter storm track intensity. Journal of Climate 15:642-658

Della-Marta PM, Pinto JG (2009) Statistical uncertainty of changes in winter storms over the North Atlantic and Europe in an ensemble of transient climate simulations. Geophysical Research Letters 36:L14703

Deser C, Tomas R, Alexander M, Lawrence D (2010) The Seasonal Atmospheric Response to Projected Arctic Sea Ice Loss in the Late 21st Century. Journal of Climate 23:333-351

Donat MG, Leckebusch GC, Pinto JG, Ulbrich U (2010) European storminess and associated circulation weather types: future changes deduced from a multi-model ensemble of GCM simulations. Climate Research 42:27-43

Donat MG, Leckebusch GC, Wild S, Ulbrich U (2011a) Future changes of European winter storm losses and extreme wind speeds in multi-model GCM and RCM simulations. Natural Hazards and Earth System Sciences 11:1351-1370

Donat MG, Renggli D, Wild S, Alexander LV, Leckebusch GC, Ulbrich U (2011b) Reanalysis suggests long-term upward trends in European storminess since 1871. Geophysical Research Letters 38:L14703

Feser F, Barcikowska M, Krueger O, Schenk F, Weisse R, Xia L (2015) Storminess over the North Atlantic and Northwestern Europe - a Review. Quarterly Journal of the Royal Meteorological Society 141:350-382

Fink AH, Brücher T, Ermert E, Krüger A, Pinto JG (2009) The European Storm Kyrill in January 2007: Synoptic Evolution and Considerations with Respect to Climate Change. Natural Hazards and Earth System Sciences 9:405-423

Fink AH, Pohle S, Pinto JG, Knippertz P (2012) Diagnosing the influence of diabatic processes on the explosive deepening of extratropical cyclones. Geophysical Research Letters 39:L07803

Gastineau G, Soden BJ (2009) Model projected changes of extreme wind events in response to global warming. Geophysical Research Letters 36:L10810

Hanna E, Cappelen J, Allan R, Jónsson T, le Blancq F, Lillington T, Hickey K (2008) New insights into North European and North Atlantic surface pressure variability, storminess and related climatic change since 1830 . Journal of Climate 21:6739-6766

Hartmann DL, Klein Tank AMG, Rusticucci M, Alexander LV, Brönnimann S, Charabi Y, Dentener FJ, Dlugokencky EJ, Easterling DR, Kaplan A, Soden BJ, Thorne PW, Wild M, Zhai PM (2013) Observations: Atmosphere and Surface. In: Stocker TF, Qin D, Plattner G-K, Tignor M, Allen SK, Boschung J, Nauels A, Xia Y, Bex V, Midgley PM (Hrsg) Climate Change 2013: The physical science basis. Contribution of Working Group I to the Fifth Assessment Report of the Intergovernmental Panel on Climate Change. Cambridge University Press, Cambridge, United Kingdom and New York, NY, USA

Harvey BJ, Shaffrey LC, Woollings TJ, Zappa G, Hodges KI (2012) How large are projected 21st century storm track changes? Geophysical Research Letters 39:L18707 
Harvey BJ, Shaffrey LC, Woollings TJ (2014) Equator-to-pole temperature differences and the extra-tropical storm track responses of the CMIP5 climate models. Climate Dynamics 43:1171-1182

Held H, Gerstengarbe FW, Pardowitz T, Pinto JG, Ulbrich U, Born K, Donat MG, Karremann MK, Leckebusch GC, Ludwig P, Nissen KM, Osterle H, Prahl BF Werner PC, Befort DJ, Burghoff O (2013) Projections of global warming induced impacts on winter storm losses in the German private household sector. Climate Change 121:195-207

Hofherr T, Kunz M (2010) Extreme wind climatology of winter storms in Germany. Climate Research 41:105-123

Hoskins BJ, Valdes PJ (1990) On the existence of storm tracks. Journal of Atmospheric Sciences 47:1854-1864

Hu Q, Tawaye Y, Feng S (2004) Variations of the Northern Hemiphere atmospheric energetics. Journal of Climate 17:1975-1986

Hueging H, Born K, Haas R, Jacob D, Pinto JG (2013) Regional changes in wind energy potential over Europe using regional climate model ensemble projections. Journal of Applied Meteorology and Climatology 52:903-917

Kirtman B, Power SB, Adedoyin JA, Boer GJ, Bojariu R, Camilloni I, Doblas-Reyes FJ, Fiore AM, Kimoto M, Meehl GA, Prather M, Sarr A, Schär C, Sutton R, van Oldenborgh GJ, Vecchi G, Wang HJ (2013) Near-term Climate Change: Projections and Predictability. In: Stocker TF, Qin D, Plattner G-K, Tignor M, Allen SK, Boschung J, Nauels A, Xia Y, Bex V, Midgley PM (Hrsg) Climate Change 2013: The physical science basis. Contribution of Working Group I to the Fifth Assessment Report of the Intergovernmental Panel on Climate Change. Cambridge University Press, Cambridge, United Kingdom and New York, NY, USA

Kovats RS, Valentini R, Bouwer LM, Georgopoulou E, Jacob D, Martin E, Rounsevell M, Soussana JF (2014) Europe. In: Barros VR, Field CB, Dokken DJ, Mastrandrea MD, Mach KJ, Bilir TE, Chatterjee M, Ebi KL, Estrada YO, Genova RC Girma B, Kissel ES, Levy AN, MacCracken S, Mastrandrea PR, White LL (Hrsg) Climate Change 2014: Impacts, Adaption and Vulnerability. Part B: Regional Aspects. Contribution of Working Group II to the Fifth Assessment Report of the Intergovernmental Panel on Climate Change. Cambridge University Press, Cambridge, United Kingdom and New York, NY, USA, S 1267-1326

Krueger O, Schenk F, Feser F, Weisse R (2013) Inconsistencies between LongTerm Trends in Storminess Derived from the 20th CR Reanalysis and Observations. Journal of Climate 26:868-874

Lambert SJ (2004) Changes in winter cyclone frequencies and strengths in transient enhanced greenhouse warming simulations using two coupled climate models. Atmosphere-Ocean 42:173-181

Matulla C, Schöner W, Alexandersson H, von Storch H, Wang XL (2008) European Storminess: Late 19th Century to Present. Climate Dynamics 31:125-130

McDonald RE (2011) Understanding the impact of climate change on Northern hemisphere extra-tropical cyclones. Climate Dynamics 37:1399-1425

Neu U, Akperov MG, Bellenbaum N, Benestad RS, Blender R, Caballero R, Cocozza A, Dacre HF, Feng Y, Fraedrich K, Grieger J, Gulev S, Hanley J, Hewson T, Inatsu M, Keay K, Kew SF, Kindem I, Leckebusch GC, Liberato MLR Lionello P, Mokhov II, Pinto JG, Raible CC, Reale M, Rudeva I, Schuster M, Simmonds I, Sinclair M, Sprenger M, Tilinina ND, Trigo IF, Ulbrich S, Ulbrich U, Wang XLL, Wernli H (2013) IMILAST: A community effort to intercompare extratropical cyclone detection and tracking algorithms. Bulletin of the American Meteorology Society 94:529-547

Pinto JG, Fröhlich EL, Leckebusch GC, Ulbrich U (2007a) Changing European storm loss potentials under modified climate conditions according to ensemble simulations of the ECHAM5/MPI-OM1 GCM. Natural Hazards and Earth System Sciences 7:165-175

Pinto JG, Ulbrich U, Leckebusch GC, Spangehl T, Reyers M, Zacharias S (2007b) Changes in storm track and cyclone activity in three SRES ensemble experiments with the ECHAM5/MPI-OM1 GCM. Climate Dynamics 29:195-121

Pinto JG, Zacharias S, Fink AH, Leckebusch GC, Ulbrich U (2009) Factors contributing to the development of extreme North Atlantic cyclones and their relationship with the NAO. Climate Dynamics 32:711-737

Pinto JG, Neuhaus CP, Leckebusch GC, Reyers M, Kerschgens M (2010) Estimation of wind storm impacts over Western Germany under future climate conditions using a statistical-dynamical downscaling approach. Tellus $A$ 62:188-201

Pinto JG, Karremann MK, Born K, Della-Marta PM, Klawa M (2012) Loss potentials associated with European windstorms under future climate conditions. Climate Research 54:1-20
Raible CC, Della-Marta PM, Schwierz C, Wernli H, Blender R (2008) Northern hemisphere extratropical cyclones: A comparison of detection and tracking methods and different reanalyses. Monthly Weather Review 136:880-897

Rauthe M, Kunz M, Kottmeier C (2010) Changes in wind gust extremes over Central Europe derived from a small ensemble of high resolution regional climate models. Meteorologische Zeitschrift 19:299-312

Reyers M, Moemken J, Pinto JG (2016) Future changes of wind energy potentials over Europe in a large CMIP5 multi-model ensemble. International Journal of Climatology. 36:783-786 doi:10.1002/joc.4382

Rockel B, Woth K (2007) Extremes of near-surface wind speed over Europe and their future changes as estimated from an ensemble of RCM simulations. Climatic Change 81:267-280

Schmidt H, von Storch H (1993) German Bight storms analyzed. Nature 365:791

Schneidereit A, Blender R, Fraedrich K, Lunkheit F (2007) Iceland climate and North Atlantic cyclones in ERA40 reanalyses. Meteorologische Zeitschrift 16:17-23

Schwierz C, Zenklusen Mutter E, Vidale PL, Wild M, Schär C, Köllner-Heck P, Bresch DN (2010) Modelling European winterwind storm losses in current and future climate. Climate Change 101:485-514

Tobin I, Vautard R, Balog I, Breon F-M, Jerez S, Ruti PM, Thais F, Vrac M, Yiou $P$ (2015) Assessing climate change impacts on European wind energy from ENSEMBLES high-resolution climate projections. Climatic Change 128:99-112

Trigo IF (2006) Climatology and interannual variability of storm tracks in the Euro-Atlantic sector: a comparison between ERA40 and NCEP/NCAR reanalyses. Climate Dynamics 26:127-143

Ulbrich U, Pinto JG, Kupfer H, Leckebusch GC, SpangehI T, Reyers M (2008) Changing Northern hemisphere storm tracks in an ensemble of IPCC climate change simulations. Journal of Climate 21:1669-1679

Ulbrich U, Leckebusch GC, Pinto JG (2009) Extra-tropical cyclones in the present and future climate: a review. Theoretical and Applied Climatology 96:117-131

Walter A, Keuler K, Jacob D, Knoche R, Block A, Kotlarski S, Mueller-Westermeier $G$, Rechid D, Ahrens W (2006) A high resolution reference data set of German wind velocity 1951-2001 and comparison with regional climate model results. Meteorologische Zeitschrift 15:585-596

Wang XL, Zwiers FW, Swail V, Feng Y (2009) Trends and variability of storminess in the Northeast Atlantic region, 18742007. Climate Dynamics 33:11791195

Wang XL, Wan H, Zwiers FW, Swail V, Compo GP, Allan RJ, Vose RS, Jourdain S, Yin $X$ (2011) Trends and low-frequency variability of storminess over western Europe 1878-2007. Climate Dynamics 37:2355-2371

Weisse R, von Storch H, Feser F (2005) Northeast Atlantic and North Sea Storminess as simulated by a regional climate model during 1958-2001 and comparison with observations. Journal of Climate 18:465-479

Woollings T, Gregory JM, Pinto JG, Reyers M, Brayshaw DJ (2012) Response of the North Atlantic storm track to climate change shaped by ocean-atmosphere coupling. Nature Geoscience 5:313-317

Yin JH (2005) A consistent poleward shift of the storm tracks in simulations of 21st century climate. Geophysical Research Letters 32:L18701

Zappa G, Shaffrey LC, Hodges KI, Sansom PG, Stephenson DB (2013) A Multimodel Assessment of Future Projections of North Atlantic and European Extratropical Cyclones in the CMIP5 Climate Models. Journal of Climate 26:5846-5862

Open Access Dieses Kapitel wird unter der Creative Commons Namensnennung 4.0 International Lizenz (http://creativecommons.org/ licenses/by/4.0/deed.de) veröffentlicht, welche die Nutzung, Vervielfältigung, Bearbeitung, Verbreitung und Wiedergabe in jeglichem Medium und Format erlaubt, sofern Sie den/die ursprünglichen Autor(en) und die Quelle ordnungsgemäß nennen, einen Link zur Creative Commons Lizenz beifügen und angeben, ob Änderungen vorgenommen wurden.

Etwaige Abbildungen oder sonstiges Drittmaterial unterliegen ebenfalls der genannten Creative Commons Lizenz, sofern sich aus der Abbildungslegende oder der Quellreferenz nichts anderes ergibt. Sofern sol- 
ches Drittmaterial nicht unter der genannten Creative Commons Lizenz steht, ist eine Vervielfältigung, Bearbeitung oder öffentliche Wiedergabe nur mit vorheriger Zustimmung des betreffenden Rechteinhabers oder auf der Grundlage einschlägiger gesetzlicher Erlaubnisvorschriften zulässig. 